DRAFT VERSION JULY 1, 2021

Preprint typeset using LATEX style emulateapj v. 5/2/11

\title{
AN ACCRETION MODEL FOR THE ANOMALOUS X-RAY PULSAR 4U 0142+61
}

\author{
J. E. TRUEMPER, K. DENNERL \\ Max-Planck-Institut für extraterrestrische Physik, Postfach 1312, 85741 Garching, Germany \\ N. D. KYLAFIS \\ University of Crete, Physics Department \& Institute of Theoretical \& Computational Physics, 71003 Heraklion, Crete, Greece \\ Foundation for Research and Technology-Hellas, 71110 Heraklion, Crete, Greece \\ Ü. ERTAN \\ Faculty of Engineering and Natural Sciences, Sabancı University, 34956, Orhanlı, Tuzla, İstanbul, Turkey \\ A. ZEZAS \\ University of Crete, Physics Department \& Institute of Theoretical \& Computational Physics, 71003 Heraklion, Crete, Greece \\ Foundation for Research and Technology-Hellas, 71110 Heraklion, Crete, Greece \\ Draft version July 1, 2021
}

\begin{abstract}
We propose that the quiescent emission of AXPs/SGRs is powered by accretion from a fallback disk, requiring magnetic dipole fields in the range $10^{12}-10^{13} \mathrm{G}$, and that the luminous hard tails of their X-ray spectra are produced by bulk-motion Comptonization in the radiative shock near the bottom of the accretion column. This radiation escapes as a fan beam, which is partly absorbed by the polar cap photosphere, heating it up to relatively high temperatures. The scattered component and the thermal emission from the polar cap form a polar beam. We test our model on the well-studied AXP 4U 0142+61, whose energy-dependent pulse profiles show double peaks, which we ascribe to the fan and polar beams. The temperature of the photosphere $(\mathrm{kT} \sim 0.4 \mathrm{keV})$ is explained by the heating effect. The scattered part forms a hard component in the polar beam. We suggest that the observed high temperatures of the polar caps of AXPs/SGRs, compared with other young neutron stars, are due to the heating by the fan beam. Using beaming functions for the fan beam and the polar beam and taking gravitational bending into account, we fit the energy-dependent pulse profiles and obtain the inclination angle and the angle between the spin axis and the magnetic dipole axis, as well as the height of the radiative shock above the stellar surface. We do not explain the high luminosity bursts, which may be produced by the classical magnetar mechanism operating in super-strong multipole fields.
\end{abstract}

Subject headings: pulsars: individual (4U 0142+61) - X-rays: stars - stars: magnetic fields - accretion disks

\section{INTRODUCTION}

Anomalous X-ray pulsars (AXPs) and Soft Gamma Ray Repeaters (SGRs) are young neutron stars with X-ray luminosities much larger than their spin-down power and long periods in the range $2-12 \mathrm{~s}$. They are widely believed to be magnetars deriving their X-ray emission from the decay of super-strong magnetic dipole fields $\left(B \gtrsim 10^{15} \mathrm{G}\right)$ (e.g. Duncan \& Thompson 1992; Thompson \& Duncan 1995). At the outset, the magnetar model was developed to explain the giant bursts and the large bursts of SGRs, which exceed the Eddington limit of neutron-star luminosities by a very large factor. If the observed spin-down of some of these sources is interpreted as the consequence of magnetic dipole braking, the resulting polar field strengths are of the order of $\gtrsim 10^{15}$, in qualitative agreement with what has been inferred from the observed luminosities (Kouveliotou et al. 1998). Later on, it was discovered that AXPs show short bursts as well, though less frequent and less energetic than SGRs, leading to the general notion that both types of sources are closely related or represent even a single class. In the magnetar picture, the steady X-ray emission of these sources, which have luminosities of typically a few times $10^{35} \mathrm{erg} \mathrm{s}^{-1}$, is thought to be caused by a twist of the magnetosphere leading to the amplification of the magnetic field and the acceleration of particles, which produce the X-ray emission. The twist is caused by rotational motions of a crust plate and has a lifetime of $\sim 1$ year (Beloborodov \& Thompson 2007). For recent reviews of the magnetar model see Woods \& Thompson (2006) and Mereghetti (2008).

An alternative energy source for the persistent and transient X-ray luminosities of AXPs and SGRs is accretion from fallback disks, first proposed by van Paradijs et al. (1995), and followed by Chatterjee et al. (2000) and Alpar (2001). This class of models was developed further in a series of papers (Ekşi \& Alpar 2003; Ertan \& Alpar 2003; Ertan \& Cheng 2004; Ertan et al. 2006; Ertan \& Çalışkan 2006; Ertan et al. 2007; Ertan \& Erkut 2008, Ertan et al. 2009). The fallbackdisk model gets support from the discovery of IR/optical radiation from two of the AXPs, 4U 0142+61 (Wang et al. 2006) and 1E 2259+586 (Kaplan et al. 2009), which has been successfully interpreted as disk emission. This optical emission is especially important, because the spectral fits at short wavelengths put an upper limit on the inner disk radius and thereby on the magnetic dipole field strength ( $\mathrm{B}<10^{13} \mathrm{G}$ ).

The fallback-disk model explains the spin-down of AXPs and SGRs by the disk-magnetosphere interaction and requires "normal" neutron-star dipole fields $\left(10^{12}-10^{13} \mathrm{G}\right)$. We note that this is in line with the recent discovery of a mag- 
netar (SGR 0418+5729) with a low magnetic dipole field $\mathrm{B}<7 \times 10^{12} \mathrm{G}$ (Rea et al. 2010; see also Alpar, Ertan \& Calışkan 2011). In addition, the model is successful in predicting the period clustering of AXPs/SGRs in the range of $2-12 \mathrm{~s}$. More recently, it was shown that the X-ray luminosity and the rotational properties, including the braking index $n=0.9 \pm 0.2$, of the so called "high-B" radio pulsar PSR J1734-3333 (Espinoza et al. 2011) can be explained by the evolution of a neutron star with a fallback disk and $B=10^{12} \mathrm{G}$ (Çalışkan et al., submitted). On the other hand, this model cannot explain the super-Eddington bursts, which are relatively rare. They are attributed to magnetar-type activities occurring in local multipole fields (star-spots).

All references quoted above, regarding the fallback-disk model, are dealing with the physics of the accretion disk and its interaction with the magnetosphere of the neutron star. A first attempt to explain the hard X-ray spectrum of the wellstudied AXP 4U 0142+61 by bulk-motion and thermal Comptonization has been presented by Truemper et al. (2010). Fig. 1 shows the excellent fit of the COMPTB model to the pulse phase averaged data from Chandra MEG and HEG at low energies and Integral ISGRI above $15 \mathrm{keV}$. In the present paper we study the energy-dependent pulse profiles and the phasedependent energy spectra, taking again 4U 0142+61 as an example.

In $\S 2$ we summarize the observational properties of $4 \mathrm{U}$ $0142+61$, in $\$ 3$ we present our model, in $\$ 4$ we interpret the phase-dependent energy spectra and infer the surface magnetic field strength, in $\S 5$ we discuss the heating of the polar cap, in $\S 6$ we compare 4U 0142+61 with low-luminosity Xray pulsars in binary systems, and in $\S 7$ we give our summary and conclusions.

\section{OBSERVATIONAL PROPERTIES OF 4 U $0142+61$}

Since its discovery by Uhuru, $4 \mathrm{U} 0142+61$ has been observed by many groups. The source has a period of $8.69 \mathrm{~s}$ and spins down with a rate of $2 \times 10^{-12} \mathrm{~s} \mathrm{~s}^{-1}$ (Dib et al. 2007). It shows a spectrum in the soft band $(0.8-10 \mathrm{keV})$ which can be fitted by a blackbody $(k T=0.42 \mathrm{keV})$ and a steep power law (photon-number spectral index $\Gamma_{s}=3.3$; e.g. Juett et al. 2002). At higher energies $(E>10 \mathrm{keV})$, a hard spectral tail $\left(\Gamma_{h}=0.93\right)$ is observed, which extends beyond 150 $\mathrm{keV}$ and contributes about one third of the total X-ray luminosity (den Hartog et al. 2008). That paper presents the most detailed data on the spectra and light curves of the persistent soft and hard X-ray emission of this source, based on observations with ASCA, XMM-Newton, RXTE, and INTEGRAL. In our present analysis, we use the data provided by den Hartog et al. (2008), since they give very detailed information on the energy-dependent pulse shapes (in particular their Fig. 9). According to this data, the luminosity of $4 \mathrm{U} 0142+61$ in the energy band $0.8-160 \mathrm{keV}$ is $\sim 4.6 \times 10^{35} \mathrm{erg} \mathrm{s}^{-1}$, assuming a distance of $3.6 \mathrm{kpc}$ (Durant \& van Kerkwijk 2006). The luminosity in the soft and the hard band is $\sim 3.2 \times 10^{35} \mathrm{erg} \mathrm{s}^{-1}$ and $\sim 1.4 \times 10^{35} \mathrm{erg} \mathrm{s}^{-1}$ respectively.

The broad-band spectrum of $4 \mathrm{U} 0142+61$ has been measured by Suzaku (Enoto et al. 2011) in the $0.4-70 \mathrm{keV}$ band, confirming the existence of a two-component spectrum. The photon index of the hard component is $\Gamma_{h}=0.9$. The Suzaku observations have the advantage that data in the hard $(>10$ $\mathrm{keV})$ and the soft $(1-10 \mathrm{keV})$ bands are taken simultaneously. The luminosities are $2.8 \times 10^{35} \mathrm{erg} \mathrm{s}^{-1}$ in the soft band $(1-10 \mathrm{keV})$ and $\sim 6.8 \times 10^{34} \mathrm{erg} \mathrm{s}^{-1}$ in the hard band $(10-70$
Table 1

Comparison between the data obtained with XMM-Newton/INTEGRAL and Suzaku

\begin{tabular}{lcc}
\hline \hline \multicolumn{1}{c}{$\begin{array}{c}\text { Energy Range } \\
(\mathrm{keV})\end{array}$} & XMM/Integral & Suzaku \\
\hline $1-10 \mathrm{keV}$ & $2.8 \times 10^{35}$ & $2.8 \times 10^{35}$ \\
$10-70 \mathrm{keV}$ & $0.64 \times 10^{35}$ & $0.64 \times 10^{35}$ \\
\hline $\mathrm{kT}_{\text {blackbody }(\mathrm{keV})}$ & $0.418 \pm 0.013^{\dagger}$ & $0.428 \pm 0.004$ \\
Power-law index $\Gamma_{\mathrm{s}}$ & $3.3 \pm 0.4^{\dagger}$ & $3.95 \pm 0.04$ \\
Power-law index $\Gamma_{\mathrm{h}}$ & 0.93 & $0.11-1.54$ \\
& & \\
\hline
\end{tabular}

Note. - $\uparrow$ We quote the Chandra data here (Juett et al. 2002), since den Hartog et al. (2008) did not perform spectral fits to the low-energy data obtained with XMMNewton.

$\mathrm{keV})$. We compared the luminosities derived from the Suzaku spectral fits with those from the combined XMM-Newton and INTEGRAL spectral fits in the same bands and we found excellent agreement (Table 1).

The same holds for the spectral parameters, except in the case of the hard power-law index derived from Suzaku, which strongly depends on the model for the soft component. In conclusion, this comparison suggests that the quiescent spectrum of the source is quite stable. It also justifies our choice to use the INTEGRAL data, which is of superior statistical quality in the hard spectral range.

Using the observational data of den Hartog et al. (2008), we have derived the observed pulsed profiles by adding the pulsed and non-pulsed components. The result is shown in Fig. 2. As noted by den Hartog et al. (2008), morphology changes are ongoing throughout the whole energy range. However, we stress that the principal structure of the pulse profiles is very simple: It mainly consists of two pulses; one is located at phase $\sim 0.1$, extending from the lowest to the highest energies $(0.8-160 \mathrm{keV})$. We call it the main pulse. The secondary pulse is located at phase $\sim 0.6$. Its relative strength decreases strongly from the lowest energy channel $(0.8-2$ $\mathrm{keV})$ to medium energies $(8-16.3 \mathrm{keV})$ and increases again in the $(20-50 \mathrm{keV})$ band.

In Fig. 3 we show the phase-dependent pulsed spectra derived from the data of den Hartog et al. (2008). The spectrum of the main pulse is taken from the phase interval $0.85-0.35$ (shown as a dashed line), while the spectrum of the secondary pulse is taken from the phase interval $0.35-0.85$ (shown as a dotted line).

\section{THE GENERAL PHYSICAL PICTURE OF OUR MODEL}

The basic picture of our model is shown schematically in Fig. 4. It is based on the model which has been developed by many authors, notably Davidson (1973), Basko \& Sunyaev (1976, hereafter BS76) and Lyubarskii \& Sunyaev (1982, hereafter LS82). The accreting matter falls freely on magnetic field lines until it is stopped in a radiative shock, where it is slowed down from a supersonic to a subsonic flow in the sinking column beneath the shock. Most of the accretion energy released in the radiative shock is transformed into hard X-ray photons by bulk-motion Comptonization (BMC, Blandford \& Payne 1981, Payne \& Blandford 1981, LS 82), which escape sideways forming a fan beam. We note that in high luminosity sources, having a large transverse optical depth in the accretion column at the height of the shock, most of the BMC photons will be downscattered before they can escape. As we discuss in section 3.2 below, due to the low 
luminosity of AXPs and SGRs, the transverse optical depth is moderate $\left(\tau_{t} \sim 5\right)$. The height of the shock above the stellar surface depends on the mass accretion rate and the cross section of the accretion column. If the fan beam is formed very close to the neutron-star surface, then about half of it escapes, while the other half - assisted by gravitational bending - hits the polar cap surrounding the accretion column. The impinging flux heats the polar cap photosphere and some part of it is scattered off by it. The scattered flux and the photospheric emission form a polar beam, which is orthogonal to the fan beam. If the radiative shock forms at a height above the neutron-star surface, then more than half of the fan beam escapes. The larger the height, the larger the fraction of the fan beam that escapes.

\subsection{Outline of the model}

In the fallback-disk model, the magnetic dipole field strength of the neutron star is in the range $10^{12}-10^{13}$. Therefore, we can employ the radiation models developed for accreting highly magnetized neutron stars in binary systems. Matter entering the magnetosphere of the neutron star at the Alfven radius falls down in the magnetic funnel provided by the dipole magnetic field of the neutron star and the flow is converted from supersonic to subsonic at a radiative shock. In the early works quoted above, a steady and homogeneous flow of matter was assumed, while Morfill et al. (1984) were the first to discuss a time-dependent flow (accretion of clumps of matter). Later on, Klein et al. (1996) studied non-steady accretion due to the formation of photon bubbles. A fully consistent description of the complicated processes governing the flow and the stopping of matter in the magnetic funnel is still lacking. Fortunately, however, the situation with AXPs and SGRs can be expected to be much simpler, due to the significantly lower accretion rate than that of most X-ray pulsars.

As discussed in $\S 2,4 \mathrm{U} 0142+61$ shows basically a doublepulse structure, with different spectra for the two components. We identify the main pulse, which extends from low energies to more than $100 \mathrm{keV}$, with the fan beam, which is radiated sideways from the column (c.f. Fig. 4a, dashed arrows), and whose high energy component $(>10 \mathrm{keV})$ is powered by BMC, taking place in the radiative shock. In the process of BMC, seed photons from below the radiative shock gain energy by back-and-forth collisions across the shock with the infalling, semi-relativistic electrons and the slowly moving, post-shock, thermal plasma beneath the shock. The thermal energy of this plasma is due to the electron recoil in the BMC process (LS82). It is well known that this first order Fermi energetization produces a power-law spectrum.

LS82 studied spectral formation due to bulk and thermal Comptonization in a radiation-dominated shock. In their model, the shock has a Rankine-Hugoniot form, and therefore the energy flux is strictly conserved. Of course, the shocks in actual X-ray pulsar accretion flows are radiative, but in practice, most of the radiation escapes through the column walls in the region downstream from the shock, and therefore the LS82 model may be an acceptable approximation for the structure of the shock itself. LS82 computed analytically the entire spectrum, not only the power law. On the other hand, the low energy $(2-10 \mathrm{keV})$ steep power law of the main pulse may be attributed to thermal Comptonization (TC) taking place in the sinking column, with seed photons produced by blackbody, bremsstrahlung, and cyclotron processes. An external source of seed photons is the radiation from the photosphere surrounding the accretion column.
The secondary pulse is attributed to photons emitted by the hot polar cap, forming a polar beam (dotted arrows in Fig. 4a. At low energies $(<2 \mathrm{keV})$, it consists of the photospheric black body-like emission and at higher energies $(>2 \mathrm{keV})$ of fan beam photons, which are scattered when they hit the photospheric plasma.

Accretion onto magnetic neutron stars has been studied extensively by Becker \& Wolff (2007; hereafter BW07). With their model, BW07 studied spectral formation in X-ray pulsars and highlighted the role of TC and BMC for the spectral formation in accretion columns. BW07 assumed a smooth flow in a cylindrical column, which is slowed down by a radiative shock. The shock is treated properly, not as a mathematical discontinuity, and it is the place where the BMC operates with seed photons produced by bremsstrahlung, cyclotron, and blackbody processes inside the column. This model predicts a fan beam. As we said above, we suggest that this process is responsible for the high energy part of the spectrum $(E>10 \mathrm{keV})$, which escapes as a fan beam. Unfortunately, BW07 did not include an external source of seed photons. Nevertheless, their model can be used to fit the spectrum of $4 \mathrm{U} 0142+61$ at high energies, which is dominated by the fan beam photons. We have done this by using the XSPEC version of the BW07 model provided by Ferrigno et al. (2009) and find that it provides a good fit to the spectrum at energies $>10 \mathrm{keV}$ (Zezas et al., in preparation). However, fitting the soft part of the spectrum requires a thermally Comptonized blackbody spectrum, which we attribute to reprocessing of the thermal radiation from the polar cap.

Another relevant model for obtaining BMC and TC spectra has been developed by Farinelli et al. (2008) for application to the spectra of low-mass X-ray binaries (LMXBs). It uses non-magnetic (Thomson) cross sections, but for our purpose it has the advantage of including an external source of seed photons. We used this model in our previous paper on $4 \mathrm{U}$ 0142+61 (Truemper et al. 2010) and obtained a good fit to the broadband X-ray spectrum of the source (see Fig. 1). The fact that it gives an excellent fit to both the soft and the hard components is significant, though the resulting fit parameters would probably be somewhat different if magnetic cross sections were used. This proviso applies to a lesser degree to the Ferrigno et al. (2009) model. For details of the application of the Ferrigno et al. (2009) and Farinelli et al. (2008) models to 4U 0142+61 and other AXPs and SGRs, we refer to Zezas et al. (in preparation).

\subsection{Physical parameters of the accretion column}

For simplicity, it is usually assumed that the accretion flow is homogeneous and the accretion funnel is cylindrical. However, for detailed comparisons between model and observations of the energy-depended pulse profiles, as we do below, this picture is too simple and qualitatively inadequate.

BS76 and LB82 have pointed out that, for a neutron star with an inclined magnetic field, the cross section of the accretion column at the neutron star surface has the shape of a thin annular arc, located at an off-center position with respect to the magnetic pole. BS76 quote a ratio of width to length of the arc of 0.025 . The off-center position of the accretion spot has been confirmed qualitatively by magneto-hydrodynamic simulations of Romanova et al. (2004), however, the width of the arc is much larger compared with the idealized model of BS76, namely $\sim 0.3-0.5$. The simulations of Romanova et al. (2004) have been done for relatively small magnetospheres, like those of accreting millisecond pulsars. Unfortunately, 
they could not be performed for the large magnetospheres of slowly spinning neutron stars with high magnetic fields due to limitations of computing power (Romanova, private communication). We assume that the picture of Romanova et al. (2004) is valid qualitatively also in our case. In Fig. 4b we sketch the footprint of the column at the neutron star surface. This geometry leads to a modulation of the radiating area (and the X-ray flux) with the rotational phase at half the neutron star period. The ratio of minimum to maximum flux is given by $d_{0} / l_{0}$. Due to the opening of the magnetic field lines, the length and the width of the accretion column scale with $r$ as $l(r)=l_{0} \times(r / R)^{3 / 2}$ and $d(r)=d_{0} \times(r / R)^{3 / 2}$.

The effective Eddington luminosity in the accretion funnel, near the neutron star surface, is Becker et al. 2012

$$
\begin{aligned}
L_{E}^{e f f}= & L_{E} \frac{l_{0} d_{0}}{4 \pi R^{2}} \frac{\sigma_{T}}{\sigma_{\|}}=\frac{G M m_{p} c}{\sigma_{T}} \frac{l_{0} d_{0}}{R^{2}} \frac{\sigma_{T}}{\sigma_{\|}} \\
= & 5.4 \times 10^{32}\left(\frac{l_{0}}{10^{4} \mathrm{~cm}}\right)\left(\frac{d_{0}}{6 \times 10^{3} \mathrm{~cm}}\right)\left(\frac{M}{1.4 M_{\odot}}\right) \times \\
& \left(\frac{12.5 \mathrm{~km}}{R}\right)^{2} \frac{\sigma_{T}}{\sigma_{\|}} \mathrm{erg} \mathrm{s}^{-1}
\end{aligned}
$$

where $L_{E}$ is the Eddington luminosity, $M$ is the mass of the neutron star, $\sigma_{T}$ is the Thomson cross section, and $\sigma_{\|}$is the average over energy cross section for photons travelling along the magnetic field.

Since the flow in the accretion funnel is supercritical, a radiative shock is established at a height $H$ above the neutron star surface (BS76, LS82, BW07). The upscattered photons in the shock escape sideways, as a fan beam, and their propagation is controlled by Thomson scattering. The transverse optical depth to electron scattering at or above the shock along the width of the funnel is

$$
\begin{aligned}
\tau_{t}=n_{e}(r) \sigma_{T} d(r) & =\frac{\dot{M}}{m_{p} v_{f f}(r) l(r) d(r)} \sigma_{T} d(r) \\
& =\frac{\dot{M}}{m_{p} v_{f f}(r) l(r)} \sigma_{T} \\
& =\frac{\dot{M} \sigma_{T}}{m_{p} v_{0} l_{0}} \frac{R}{r},
\end{aligned}
$$

where $n_{e}(r)$ is the electron density, and $v_{f f}(r)=$ $v_{0}(R / r)^{1 / 2}$ the free-fall speed, $v_{0}=(2 G M / R)^{1 / 2} \approx$ $0.6 c\left(M / M_{0}\right)^{1 / 2}(12.5 \mathrm{~km} / R)^{1 / 2}$, and $\dot{M}$ is the mass accretion rate. The transverse optical depth cannot be large, as it is in X-ray pulsars, because the BMC photons would be downscattered before they escaped. On the other hand, it cannot be too small, because BMC would be inefficient. In order to have a transverse optical depth of order a few, for accretion rates of the order of $10^{15} \mathrm{~g} \mathrm{~s}^{-1}$, the length $l_{0}$ must be of the order of $10^{4} \mathrm{~cm}$.

The analytic solution for the spectrum produced at the shock, that was found by LS82, provides for a high-energy power-law spectrum with index $\Gamma_{h} \sim 0.9$. Using a Monte Carlo code (Kylafis \& Truemper, in preparation), we have obtained a similar spectrum, if the transverse Thomson optical depth at the shock is $\tau_{t}(R+H) \sim 5$. Using eq. (2), this implies that for $H<R$ the height of the shock is given approximately by

$$
H \sim\left(\frac{\dot{M} \sigma_{T}}{5 m_{p} v_{0} l_{0}}-1\right) R \sim 0.2 R,
$$

where we have used $\dot{M}=3 \times 10^{15} \mathrm{~g} \mathrm{~s}^{-1}$ and $l_{0}=10^{4} \mathrm{~cm}$. It is clear from the above, that despite the low luminosity of AXPs and SGRs, the radiative shock may be located at some height, depending on the accretion rate, the footprint of the accretion column, and the gravitational field.

For completeness, we mention that in the polar direction (i.e., parallel to the magnetic field), the Thomson optical depth of the accretion column is

$$
\tau_{p}=\frac{2}{3} \tau_{t}(R) \frac{R}{d_{0}}>>\tau_{t}(R),
$$

\subsection{Modeling the pulse shapes of $4 U 0142+61$}

As already noted above, after almost forty years of theoretical efforts, there is no fully selfconsistent description yet of the radiation from an accretion column. This is true in particular for the angle- and energy-dependence of the emission. The most advanced approach is the one by BW07, which describes the spectral formation in terms of TC and BMC, but uses angle averaged scattering cross sections, which are not suited to derive angular distributions. We therefore use a pragmatic approach and introduce beaming functions to describe the angular dependence of the different beams. This method has been used widely for the analysis of X-ray pulsar profiles (e.g., Leahy 1990, 2003; Kraus 2001).

To model the observed pulse profiles, we assume that the neutron star has a centered magnetic dipole. Thus, the fan beam, which escapes sideways from the column, and the polar beam are orthogonal. The beaming functions are given by $f \sin ^{m} \theta$ and $p \cos ^{n} \theta$, for the fan beam and the polar beam, respectively. Here $\theta$ is the polar angle, i.e. the angle between the direction of photon propagation and the magnetic dipole axis. The amplitudes $f$ and $p$ and the exponents $m$ and $n$ are free parameters, which may be different for different energy intervals. Note that $m=1$ or $n=1$ corresponds to the case of isotropic emission, e.g. blackbody emission. The ansatz for the beaming function is a generalized version of the angular distributions of photons presented by Nagel (1981) and Meszaros \& Nagel (1985a,b) for columns and slabs, which can be described by $\sin ^{m} \theta$ and $\cos ^{n} \theta$, respectively, with $m$ and $n \leq 1.5$, except for an energy interval around the cyclotron resonance. In that work, the full angle- and energy-dependent magnetic scattering cross sections, including effects of vacuum polarization were used, assuming a homogeneous temperature and density distribution in the radiating slab or column. However, since in the outer layers of a slab or a column the plasma temperature will drop towards the surface, photons escaping perpendicular to the surface will escape from deeper layers than photons escaping in other directions. This leads to a limb-darkening effect, which makes the beaming patterns narrower compared with those of Nagel (1981) and Meszaros \& Nagel (1985a,b). Thus, the expected exponents of the beaming will be significantly larger than 1.5.

A model with orthogonal fan and polar beams will lead to symmetric pulse profiles with double pulses separated by 0.5 in phase, in disagreement with the observed pulse profiles, which show asymmetries and slightly different pulse separations. To account for these effects we assume, as discussed in Section 3.2, that the accretion column has a non-cylindrical cross section with major axis $l_{0}$ and minor axis $d_{0}$ (c.f. Fig 
4b).

To account for gravitational bending, we use the expression derived by Beloborodov (2002). For a photon emitted near the neutron star at an angle $\theta$ with respect to the radial direction, the angle $\theta+\delta$, at which it arrives at infinity, is given by

$$
1-\cos (\theta+\delta)=\frac{1-\cos \theta}{1-\frac{R_{s}}{R}},
$$

where $R$ is the radius of the neutron star and $R_{s}=2 G M / R c^{2}$, is its Schwarzschild radius. This equation provides a very good approximation in a Schwarzschild metric for $R / R_{s}>2$. We assume $M=1.4 M_{\odot}$ and $R=12.5 \mathrm{~km}$, viz. $R / R_{s}=3$. An important parameter is the height $H$ of the radiative shock above the neutron star surface. In the following we discuss three cases: $H=0, H=2 \mathrm{~km}$, and $H=0.5 R$.

a) $H=0$ : This choice is motivated by the general view that at low accretion rate the matter is stopped by the radiative shock at a position close to the stellar surface. The fitting parameters are: the inclination $i$ of the system, the angle $\alpha$ between the rotation axis and the magnetic dipole axis, the axis ratio $d_{0} / l_{0}$, the azimuthal angle $\phi$ of the long axis, phase zero of the pulse profile, the exponents $m$ and $n$ of the beaming functions for the fan and the polar beam, and the normalizations $f$ and $p$ of the fan beam component and the polar beam component, respectively. We remark that the first five parameters ( $i, \alpha, d_{0} / l_{0}, \phi$, and phase zero) are fitted jointly for all energy bands, while the others are fitted for each energy band separately.

The fit is done by a hierarchical grid search. In an outer loop the global parameters are scanned in a five-dimensional grid. In an inner loop the two exponents and the flux ratio between the fan and polar beam are scanned in a three-dimensional grid, for each combination of the global parameters of the outer grid. For each candidate pulse profile, the overall normalization of the flux is directly determined by the ratio of the mean flux of that profile and the measured profile in the corresponding energy range.

This procedure is applied several times, starting with a coarse grid covering a wide range of parameters. This grid is subsequently refined by a denser grid around the solution found in the preceding step. We use the $\chi^{2}$ statistics as the criterion for the agreement between the observed and calculated pulse profiles. In order to avoid that the solution is dominated by the low energy profiles, which have the best statistical quality, we add systematic errors to the statistical errors, individually for each energy band, so that formally acceptable fits are obtained. This method allows us also to quantify to which accuracy our model is capable of reproducing the observed pulse profiles. We find systematic errors of $2.1 \%$, $1.7 \%, 4.4 \%, 3.5 \%, 3.5 \%$ for the pulse profiles of the corresponding ranges $0.8-2.0 \mathrm{keV}, 2-4 \mathrm{keV}, 4-8 \mathrm{keV}, 8-16.3$ $\mathrm{keV}, 20-50 \mathrm{keV}$ (for the $50-160 \mathrm{keV}$ band, no additional error is required). We then estimate the uncertainty in the model parameters by subsequently varying the individual parameters in both directions (while keeping the other parameters fixed at the best-fit values), until the $\chi^{2}$ increases by 1 , which corresponds to the $68 \%$ confidence level for 1 interesting parameter. Fig. 5a shows the model fit to the observed light curves. The inclination is $i=42.1 \pm 0.4$ degrees, the angle between the dipole with respect to the spin axis $\alpha=19 \pm 0.9$ degrees and the axis ratio of the accretion column is $d_{0} / l_{0}=0.69 \pm 0.01$, where the errors are $1 \sigma$.
Our model gives us information about the full beaming geometry, and thus allows us to determine the "intrinsic luminosities" of the different beams. This is a major advantage compared to analysis methods which are purely based on the observed flux, i.e., the flux which we sample along the line of sight during the rotation of the neutron star. Depending on the geometrical circumstances, the observed flux may not be representative for the $X$-ray emission of the neutron star. In the following we utilize the information about the full beaming geometry to compute which fraction of the intrinsic fan beam luminosity irradiates the neutron star and compare this fraction with the intrinsic luminosity of the polar beam. For clarity, we focus in the following on the properties of one pole (due to the symmetry of our model, the properties of the other pole are identical).

According to the $H=0$ model, the intrinsic $0.8-160 \mathrm{keV}$ luminosity of the fan beam is $4.4 \times 10^{35} \mathrm{erg} \mathrm{s}^{-1}$, which includes the fraction which falls onto the neutron star and the one which escapes. The intrinsic luminosity of the polar beam is $0.7 \times 10^{35} \mathrm{erg} \mathrm{s}^{-1}$, viz. $15 \%$ of the energy of the intrinsic fan beam. The expected fraction of the total luminosity of the fan beam hitting the neutron star is $50 \%$. Thus the neutron star reemits only a fraction $15 / 50=30 \%$ of the incident flux, while the rest disappears, e.g. in the neutron-star crust. As this seems quite unlikely, we investigated an alternative scenario, where the radiative shock is not located at the stellar surface, but at some height $H$ above it. In this case, the neutron star intercepts less than half of the incident fan beam flux.

b) $H=2 \mathrm{~km}$ : In order to compute which fraction of the total fan beam luminosity reaches the neutron star for the case $H>0$, it is necessary to know the trajectories of the emitted photons in the presence of gravitational bending. Such trajectories are found in the work of Leahy (2003) for a neutron star radius $R=12.5 \mathrm{~km}$. He shows that X-rays emitted from the accretion column at a height $H$ reach the neutron star if they are emitted at zenith angles $\theta>\theta_{i}(H)$, while the rest escapes. Implementing this condition into the model and assuming $H=2 \mathrm{~km}$, the solution is shown in Fig. 5b. For this model, we find systematic errors of $2.0 \%, 3.1 \%, 7.1 \%, 6.1 \%$, $7.5 \%$ for the pulse profiles of the corresponding ranges $0.8-$ $2.0 \mathrm{keV}, 2-4 \mathrm{keV}, 4-8 \mathrm{keV}, 8-16.3 \mathrm{keV}, 20-50 \mathrm{keV}$ (for the $50-160 \mathrm{keV}$ band, no additional error is required). The inclination is $i=59.6 \pm 2.4$ degrees, the angle between the dipole and the spin axis $\alpha=31.2 \pm 1.7$ degrees, and the axis ratio of the accretion column is $d_{0} / l_{0}=4 / 5$. The intrinsic 0.8 $-160 \mathrm{keV}$ luminosity of the fan beam is $1.8 \times 10^{35} \mathrm{erg} \mathrm{s}^{-1}$ and that of the polar beam is $0.3 \times 10^{35} \mathrm{erg} \mathrm{s}^{-1}$. Thus the ratio of the luminosities is again $15 \%$. However, the fraction of the total luminosity of the fan beam which reaches the neutron star is now smaller. Its exact value depends on the exponent $m$. For a typical $m=4$, it is about $20 \%$. In this case, an X-ray albedo of $15 / 20=75 \%$ would be required for the neutron star, which looks more plausible than the $30 \%$ found for $H=0$.

c) $H=0.5 R$ : We also investigated the case $\mathrm{H}=6.25 \mathrm{~km}$ and obtained fit results $\left(i=55.0 \mathrm{deg}, \alpha=30.5 \mathrm{deg}, d_{0} / l_{0}=\right.$ $4 / 5)$, which are very similar to those for $H=2 \mathrm{~km}$. This is a consequence of the fact that the effect of gravitational bending becomes less important with increasing height. Compared to $H=2 \mathrm{~km}$, the intrinsic luminosity of the fan beam increases slightly (by less than 3\%), while the polar beam luminosity stays the same, so that the ratio is again $\sim 15 \%$. At $H=0.5 R$, however, the neutron star intercepts only $\sim 5 \%$ of the incident fan beam flux, so that the fan beam could power only $5 / 15=$ 
$1 / 3$ of the polar beam luminosity.

The fact that the fit results are rather insensitive to $H$, for $H$ between $2 \mathrm{~km}$ and $6.25 \mathrm{~km}$, allows us to compute the maximum value of $H$ in a straightforward way, by determining the height at which the neutron star would intercept $15 \%$ of the fan beam luminosity. This value depends on the exponent $m$ and decreases with increasing $m$. For representative values $m=2$ and 3 , it is $\sim 3.5 \mathrm{~km}$ and $\sim 2.8 \mathrm{~km}$, respectively. We note that the upper limit of $\sim 3.5 \mathrm{~km}$ or $0.3 \mathrm{R}$, which we find for the height of the radiative shock, is consistent with the height estimate $(\sim 2.5 \mathrm{~km})$ presented in section 3.2.

\section{THE PHASE-DEPENDENT SPECTRA - AN ESTIMATE OF THE MAGNETIC DIPOLE FIELD OF AXP 4U 0142+61?}

As already discussed in Sections 3.2 and 3.4 due to the beaming geometry and enhancement by gravitational bending, a substantial part of the fan beam hits the polar cap photosphere and will heat it up. A fraction of the infalling photons will be scattered, leading to a reflected beam emitted in the polar direction. The reflection coefficient depends on photon energy and reaches a maximum at the local cyclotron frequency. Thus, one expects that the intensity ratio of the polar to the fan beam increases towards the cyclotron energy. Such an increase is indeed observed (Fig. 3). At $\sim 60 \mathrm{keV}$, the polar beam reaches its maximum, followed by a decline or cutoff beyond $\sim 70 \mathrm{keV}$. Thus, the corresponding cyclotron energy is $\sim 60 \mathrm{keV}$, implying a polar magnetic field of $B \sim 5 \times 10^{12}(1+z) \mathrm{G}$, where $z$ is the gravitational redshift. We note that the rather large apparent width of this feature may be due to the variation of the magnetic dipole field over the polar cap photosphere, which according to observations has a radius of $\sim 8 \mathrm{~km}$ (White et al. 1996; Israel et al. 1999; Juett et al. 2002). Clearly, a confirmation of this spectral feature and a more accurate determination of its position and width in energy will allow us to further test the accuracy of these estimates.

\section{HEATING OF THE POLAR CAP}

It is well known that the polar caps of AXPs/SGRs show significantly higher photospheric temperatures compared with other relatively young neutron stars, like radio pulsars (e.g. Aguilera et al. 2008). It is evident that our model provides a natural explanation for this observational fact, since it predicts a strong illumination of the photosphere surrounding the accretion column by the fan beam. The efficiency of this process is increased by gravitational bending.

Because of the relatively high threshold $(>0.8 \mathrm{keV})$, the XMM-Newton data of den Hartog et al. (2008) taken in 2003 and 2004 do not contain information on the blackbody component. The Chandra MEG and HEG observations of 4U 0142+61 on 23 May 2001 (Juett et al. 2002) have measured the spectrum at lower energies $(0.5-10 \mathrm{keV})$ which can be fitted by a blackbody $\left(k T=0.418 \pm 0.013 \mathrm{keV}, L_{b b}=\right.$ $\left.5.7 \times 10^{34}(d / 3.6 \mathrm{kpc})^{2} \mathrm{erg} \mathrm{s}^{-1}\right)$ and a power-law component with a photon index $\Gamma_{s}=3.3$. The observed energy flux in the $0.8-2 \mathrm{keV}$ interval is $47.5 \mathrm{eV} \mathrm{cm}^{-2} \mathrm{~s}^{-1}$. In our data, based on the XMM-Newton observations, the corresponding flux is $87.6 \mathrm{eV} \mathrm{cm}^{-2} \mathrm{~s}^{-1}$. The difference may be due to source variability and/or systematic errors. Assuming that the ratio of blackbody and power law energy flux (which is 1) is the same in both data sets we find a blackbody luminosity $L_{b b}=10^{35} \mathrm{erg}$ $\mathrm{s}^{-1}$ expected for the XMM-Newton spectrum.

The blackbody luminosity of $4 \mathrm{U} 0142+61$ can also be derived from data presented by Enoto et al. (2011). They fit two blackbodies to the soft component with $k T_{1}=0.337 \mathrm{keV}$, $R_{1}=13.2 \mathrm{~km}$ and $k T_{2}=0.633 \mathrm{keV}, R_{2}=2.3 \mathrm{~km}$. The corresponding blackbody luminosities are $L_{1}=7.25 \times 10^{34} \mathrm{erg}$ $\mathrm{s}^{-1}$ and $L_{2}=2.69 \times 10^{34} \mathrm{erg} \mathrm{s}^{-1}$. Thus, the total blackbody luminosity is $L_{b b}=10^{35} \mathrm{erg} \mathrm{s}^{-1}$, in good agreement with the value derived from the Chandra data for the time of the XMMNewton observations. This result, together with the agreement between the XMM-Newton data and the Suzaku data presented in table 1, shows that the observational data are consistent.

Traditionally, the soft component of AXPs and SGRs has been fitted by two different models: a) Blackbody plus power law $(\mathrm{BB}+\mathrm{PL})$ or b) double blackbody $(\mathrm{dBB})$. Halpern \& Gotthelf (2004) discussed the merits of both models and supported interpretation $b$. Our results shed new light on this question. In our model, the power-law component can be attributed to thermal Comptonization, taking place in the hot plasma below the radiative shock. However, a natural consequence of our model is that the temperature falls off on the polar cap with the distance from the accretion column, which would be consistent with the double blackbody fit discussed by Enoto et al. (2011). It seems that at present the observational data and the model predictions are not good enough to distinguish between these alternatives or their combination.

\section{COMPARISON WITH X-RAY BINARY NEUTRON STARS}

It is an interesting question why these AXPs/SGRs have hard X-ray tails up to $100 \mathrm{keV}$ or more (Enoto et al. 2010), while in general the accreting binary neutron stars show an exponential cutoff at energies of $10-20 \mathrm{keV}$. The major difference between the two classes is that AXPs/SGRs have luminosities which are about two or three orders of magnitude lower than typical X-ray binaries like Her X-1. To our knowledge, the only known accreting X-ray pulsars in binary system, which come close to $4 \mathrm{U} 0142+61$ with respect to luminosity and magnetic field strength, are 4U 0352+309 (X Persei) and 4U 2206+ 54, which we discuss below. Although there are a few additional sources that could be considered as members of this class of objects (e.g. 4U 1145-61 and $4 \mathrm{U}$ 1258-61), the scarce available hard X-ray data on them do not allow us to investigate the nature (and significance in the case of $4 \mathrm{U}$ 1258-61) of their hard X-ray emission at the level of detail that is possible for X Persei and 4U 2206+ 54 .

4U 0352+309 (X-Per): According to BeppoSAX observations of di Salvo et al. (1998), this source has a luminosity of $2.4 \times 10^{34} \mathrm{erg} \mathrm{s}^{-1}$, with half of the luminosity in the $0.1-10$ $\mathrm{keV}$ band and the other half in the $10-100 \mathrm{keV}$ band. A crude estimate derived from the spin-up rate gives a magnetic dipole field for $4 \mathrm{U} 0352+309$ of $B \sim 2.5 \times 10^{12}(1+z) \mathrm{G}$ (Ghosh \& Lamb 1979). The detection of a cyclotron line at $\sim 29 \mathrm{keV}$, implying a magnetic field strength of $B \sim 2.5 \times 10^{12}(1+z) \mathrm{G}$, has been reported by Coburn et al. (2001). Thus, the magnetic field strength of 4U $0352+309$ (X Persei) is comparable with that expected for fallback-disk accretors. The spectrum of the source exhibits a soft thermal component below $20 \mathrm{keV}$ and a distinct hard tail (di Salvo et al. 1998). The photon number spectral index of the hard component between 30 and 70 $\mathrm{keV}$ is $\Gamma_{h} \sim 1.35$, while for the hard tail of $4 \mathrm{U} 0142+61$ it is $\Gamma_{h} \sim 0.93$ (den Hartog et al. 2008, see also Truemper et al. 2010) or $\Gamma_{h} \sim 0.9$ (Enoto et al. 2011). Thus, this source has very similar spectral characteristics as 4U 0142+61. Recently, Lutovinov et al. (2012) described the activity of the source during 2001-2011 and reported several consecutive outbursts 
during which the luminosities increased up to $1.2 \times 10^{35} \mathrm{erg}$ $\mathrm{s}^{-1}$ and a spectrum showing a distinct hard X-ray tail up to energies of $\sim 160 \mathrm{keV}$. The cyclotron line at $29 \mathrm{keV}$ is confirmed.

We suggest that the power-law tail of $4 \mathrm{U} 0352+309$ is due to the same mechanism operating in $4 \mathrm{U} 0142+61$, namely bulkmotion Comptonization. ${ }^{1}$

$4 \mathrm{U} 2206+54$ : This is a pulsar showing very slow pulsations $(P=5560 \mathrm{~s}$, Reig et al. 2009) accreting matter from the stellar wind of an O-type companion. A detailed analysis of the magnetorotational evolution requires a magnetic field of the neutron star $B>5 \times 10^{13} \mathrm{G}$ (Ikhsanov \& Beskrovnaya 2011). This is in conflict with a possible cyclotron resonance scattering feature at $\sim 30 \mathrm{keV}$ claimed by Torrejon et al. (2004), Masetti et al. (2004), Blay et al. (2005), and Wang (2009), which would indicate a magnetic field strength of $\sim 3.3 \times 10^{12} \mathrm{G}$, but which could not be detected by other observations (Reig et al. 2009; Wang 2010). At any rate, the dipole magnetic field must be large, although it will be smaller than the magnetic fields usually assigned to magnetars (Reig et al. 2012). The source shows intensity variations of a factor 5 in the soft band $(2-10 \mathrm{keV})$, ranging from 1.5 to $8 \times 10^{-3}$ photons $\mathrm{cm}^{-2} \mathrm{~s}^{-1}$. The average luminosity in the $2-10 \mathrm{keV}$ band is $1.5 \times 10^{35} \mathrm{erg} \mathrm{s}^{-1}$ for a distance of $2.6 \mathrm{kpc}$ (e.g. Reig et al. 2012). Thus the luminosity and the magnetic dipole strength are comparable with those of AXPs/SGRs.

But there are also other similarities: 1) The soft spectrum can be modeled by a blackbody and a power-law component. The blackbody radius is $\sim 2.6 \mathrm{~km}$ which is consistent with the size of a hot polar cap of the neutron star (Reig et al. 2012). 2) At energies $>20 \mathrm{keV}$ a power-law spectrum is observed which has been measured up to energies of more than 100 $\mathrm{keV}$ whose steepness depends on the intensity level. At low luminosity levels $\left(\gtrsim 10^{34} \mathrm{erg} \mathrm{s}^{-1}\right.$ ), the source shows a hard power-law tail at energies $>20 \mathrm{keV}$ ranging up to more than $100 \mathrm{keV}$ with a slope $\Gamma_{h} \sim 2$ (Torrejon et al. 2004; Masetti et al. 2004; Reig et al. 2009). At higher luminosity levels $\left(\sim 2.7 \times 10^{35} \mathrm{erg} \mathrm{s}^{-1}\right)$ the hard spectrum becomes significantly steeper with $\Gamma_{h} \sim 3$ (Reig et al. 2012). Actually its overall shape approaches that of high luminosity pulsars like Her X-1 which shows a spectral cut-off at $\sim 20 \mathrm{keV}$.

Concluding this discussion, we note that the similarity in the spectral behavior of $4 \mathrm{U} 0352+309$ and $4 \mathrm{U} 2206+54 \mathrm{com}-$ pared with AXPs/SGRs on the one hand and the difference from the majority of the high luminosity binary X-ray sources on the other, supports our view (see section 3.2) that in the luminosity range around $10^{35} \mathrm{erg} \mathrm{s}^{-1}$ the transverse optical depth of the accretion column $(\tau \sim 5)$ is favorable for the production and escape of BMC photons.

\section{SUMMARY AND CONCLUSIONS}

1. We have shown in a semi-quantitative way that bulkmotion/thermal Comptonization in an accretion column, formed by a dipole magnetic field of strength $B \sim 10^{13} \mathrm{G}$, describes well not only the soft and hard Xray spectra but also the phase-dependent energy spectra of AXP 4U 0142+61.

\footnotetext{
${ }^{1}$ We note, that during the refereeing process of our work, Doroshenko et al. (2012) published a paper in which they analyzed the spectrum of $4 \mathrm{U}$ $0352+309$ observed with Integral and fitted it with a semi-phenomenological thermal and bulk-motion Comptonization model for the soft and hard component, respectively.
}

2. Furthermore, we have shown in a quantitative way that the model explains naturally the observed pulse profiles in all X-ray bands. The energy-dependent pulse profiles and their constancy over long periods of time constitute significant observational constraints for the proposed models. Our model explains them in a simple and natural way by the formation of two "beams", one perpendicular to the accretion column (main pulse) and another parallel to it (secondary pulse).

3. Our beaming model takes the gravitational bending and a noncircular shape of the accretion column into account, allowing us to determine both the angle $a$ between the spin axis and the magnetic dipole axis, and the inclination $i$ of the spin axis with respect to the line of sight. Assuming that the radiative shock is located very close to the stellar surface $(H=0)$, we find a good fit with $i \sim 40$ degrees and $\alpha \sim 20$ degrees. However, the ratio of polar to fan beam flux is too small, as only $30 \%$ of the impinging fan beam flux is converted into the scattered and thermal albedo. For a height of the radiative shock of $H=2 \mathrm{~km}$, we find $i \sim 60$ degrees, $\alpha \sim 30$ degrees, and a more reasonable value for the albedo, which is $\sim 75 \%$. We conclude that our fits provide evidence for a height $H$ of the order of at most 3.5 $\mathrm{km}$.

4. The Suzaku observations (Enoto et al. 2010) have provided a homogeneous set of spectra of 2 SGRs and 5 AXPs, showing that the fluxes of the soft $F_{s}$ and the hard $F_{h}$ spectral components are roughly equal, with variations of a factor 3 . In view of the similarity of their broad-band spectra, it is no surprise that accretion models with BB, TC, and BMC components can fit their broad-band $(0.5-200 \mathrm{keV})$ spectra (Zezas et al., in preparation). The observed ratio of these observed fluxes $F_{h} / F_{s}$ will depend on the range of polar angles $\theta$ swept by the line of sight during one revolution. In addition, one expects in our model that the hard luminosity produced by BMC correlates with the accretion rate, and for disk accretion the latter is expected to correlate with the period derivative $\dot{P}$. Thus, one expects a correlation of $F_{h} / F_{s}$ with $\dot{P}$. This is in agreement with the Suzaku data which show a correlation of $F_{h} / F_{s}$ with $\dot{P}$.

5. We suggest that the formation of hard X-ray spectral tails in accretion columns depends crucially on the optical depth of the accretion column, which is expected to correlate with X-ray luminosity. For high luminosity sources, the transverse optical depth $\tau_{t}$ of the accretion column is high, and the high energy photons produced by BMC are thermalized before they escape. In AXPs and SGRs, having luminosities a factor of $\sim 100$ less, the optical depth across the column is large enough to ensure efficient upscattering of photons to high energies $(E \gtrsim 100 \mathrm{keV})$ and low enough to allow their escape before thermalization. We suggest that this requires $\tau_{t} \sim 5$.

6. The fact that the accreting X-ray binary $4 \mathrm{U} 0352+309$ (X Per), having a luminosity about a factor of 10 smaller than $4 \mathrm{U} 0142+61$, shows a luminous hard tail strongly supports our model. In this case, the optical 
depth may be expected to be even smaller, which would explain the relatively low cutoff of the hard component ( $\sim 65 \mathrm{keV})$. The low luminosity accreting neutron star 4 U 2203+54 has a hard tail as well showing a significant steepening of its hard spectrum at transitions from low to high luminosities, which is in line with the conclusion of point 5 .

7. It is well known that the polar caps of AXPs/SGRs show significantly higher photospheric temperatures compared with other relatively young neutron stars, like radio pulsars or X-ray dim neutron stars (XDINs) (e.g. Aguilera et al. 2008). Evidently, our model provides a natural explanation for this observational fact, since the strong illumination of the photosphere by the fan beam acts as an additional heat source. The efficiency of this process is increased by gravitational bending. The heat influx into the photosphere will depend primarily on the power of the fan beam and its width, as well as on the gravity of the neutron star.

8. We propose that the spectral bump of the secondary pulse (which corresponds to the polar beam) is caused by cyclotron resonance reflection of the fan beam by the magnetized photosphere. This leads to magnetic field strength of $B \sim 5 \times 10^{12}(1+z) \mathrm{G}$, which falls in the range of dipole magnetic fields $\left(10^{12}-10^{13}\right.$ G) attributed to AXPs/SGRs in the framework of the fallback-disk scenario. Support for the above proposal is the steepness of the polar beam spectrum in the $10-$ $60 \mathrm{keV}$ range.

9. We stress that the few giant bursts observed from SGRs cannot be explained by any accretion process. The same is true for other bursts with large super-Eddington luminosities. They are most likely caused by processes taking place at the stellar surface, e.g. conversion of super-strong magnetic fields $\left(B \sim 10^{15} \mathrm{G}\right)$ into radiation triggered by crustal shifts, as discussed in the classical magnetar literature (e.g. Thomson \& Duncan 1995). Our analysis suggests that these events do not take place in the dipole field, but in localized multipole fields. This situation is qualitatively similar to that of the Sun, which shows flare activities in sunspot fields, which are larger than the solar dipole field by at least two orders of magnitude.

10. Finally, we note that the kind of analysis presented in this paper - applied to other AXPs/SGRs observed with sufficiently large photon statistics - can be used to study in detail the radiative properties of accretion columns and to narrow the gap between observations and theoretical models in this important field of Astrophysics.

This research has been supported in part by EU Marie Curie project no. 39965, EU REGPOT project number 206469 and by EU FPG Marie Curie Transfer of Knowledge Project ASTRONS, MKTD-CT-2006-042722. Ü.E. acknowledges research support from TÜBITAK (The Scientific and Technical Research Council of Turkey) through grant 110T243 and support from the Sabanc1 University Astrophysics and Space Forum. We also thank Xiao-Ling Zhang (MPE) for her help with a search of the ISGRI data bank.
Facilities: INTEGRAL, XMM-Newton.

\section{REFERENCES}

Alpar, M. A. 2001, ApJ, 554, 1245

Alpar, M. A., Ertan, Ü., \& Caliscan, S. 2011, ApJ, 732, L4

Aguilera, D. N., Pons, J., \& Miralles, J. A. 2008, ApJ, 673, 167

Basko, M. M., \& Sunyaev, R. A. 1976, MNRAS, 175, 395 (BS76)

Becker, P. A., Klochkov, D., Schönherr, G., Nishimura, O., Ferrigno, C., Caballero, I., Kretschmar, P., Wolff, M. T., Wilms, J., \& Staubert, R. 2012, A\&A, 544, 123

Becker, P. A., \& Wolff, M. T. 2007, ApJ, 654, 435 (BW07)

Beloborodov, A. M. 2002, ApJ, 566, 85

Beloborodov, A. M., \& Thompson, C. 2007, ApJ, 657, 967

Blandford, R. D., \& Payne, D. G., 1981, MNRAS, 194, 1041

Blay, P., Ribó, M., Negueruela, I., Torrejón, J. M., Reig, P., Camero, A., Mirabel, I. F., Reglero, V. 2005, A\&A, 438, 963

Chatterjee, P., Hernquist, L., \& Narayan, R. 2000, ApJ, 534, 373

Coburn, W., Heindl, W. A., Gruber, D. E., Rothschild, R. E., Staubert, R., Wilms, J., \& Kreykenbohm, I. 2001, ApJ, 552, 738

Davidson, K. 1973, Nature, 246, 1

den Hartog, P. R., Kuiper, L., Hermsen, W., et al. 2008, A\&A, 489, 245

di Salvo, T., Burderi, L., Robba, N. R., \& Guainazzi, M. 1998, ApJ, 509, 897

Dib, R., Kaspi, V.M., \& Gavriil, F.P. 2007, ApJ, 666, 1152

Doroshenko, V., Santagelo, A., Kreykenbohm, I., \& Dorshenko, R. 2012,

A\&A, 540, L1

Duncan, R. A., \& Thompson, C. 1992, ApJ, 392, 9

Durant, M., \& van Kerkwijk, M.H. 2006, ApJ, 652,576

Ekşi, K. Y., \& Alpar, M. A. 2003, ApJ, 599, 450

Enoto, T., Nakazawa, K., Makishima, K. et al. 2010, ApJ, 722, 162

Enoto, T., Makishima, K., Nakazawa, K., Kokubun, M., Kawaharada, M., Kotoku, J., \& Shibazaki, N. 2011, PASJ, 63, 387

Ertan, Ü., \& Alpar, M. A. 2003, ApJ, 593, 93

Ertan, Ü., \& Çalışkan, Ş. 2006, ApJ, 649, 87

Ertan, Ü, \& Cheng, K. S. 2004, ApJ, 605, 840

Ertan, Ü., Ekşi, K. Y., Erkut, M. H., \& Alpar, M. A. 2009, ApJ, 702, 1309

Ertan, Ü., Erkut, M. H., Ekşi, K. Y., \& Alpar, M. A. 2007, ApJ, 657, 441

Ertan, Ü., \& Erkut, M. H. 2008, ApJ, 673, 1062

Ertan, Ü., Göğüş, E., \& Alpar, M.A. 2006, ApJ, 640, 435

Espinoza, C. M., Lyne, A. G., Kramer, M., Manchester, R. N., \& Kaspi,V. M. 2011, ApJ, 741, L13

Farinelli, R., Titarchuk, L., Paizis, A., \& Frontera, F., 2008, ApJ, 680, 602

Ferrigno, C., Becker, P. A., Segreto, A., Mineo, T., \& Santangelo, A. 2009,

A\&A, 498, 825

Ghosh, P., \& Lamb, F. K. 1979, ApJ, 234, 296

Halpern, J. P. \& Gotthelf, E. V. 2004, ApJ, 632, 1075

Ikhsanov, N.R., \& Beskrovnaya, N.G. 2011, in High-Energy Emission from Pulsars and their Systems, Astrophysics and Space Science Proceedings, p. 341, Springer-Verlag, Berlin, Heidelberg

Israel, G. L., Angelini, L., Burderi, L., et al. 1999, Nuclear Physics B, 69, 141

Juett, A. M., Marshall, H. L., Chakrabarty, D., \& Schulz, N. S. 2002, ApJ, 568,31

Kaplan, D. L., Chakrabarty, D., Wang, Z., \& Wachter, S. 2009, ApJ, 700, 149

Klein, R. L., Arons, J., Jernigan, G., \& Hsu, J. 1996, ApJ, 457, L85

Kouveliotou, C., Dieters, S., Strohmayer, T., et al. 1998, Nature, 393, 235

Kraus, U. 2001, ApJ, 563, 289

Leahy, D. A. 1990, MNRAS, 242, 188

Leahy, D.A. 2003, ApJ, 596, 1131

Lyubarski, Yu. E., \& Syunyaev, R. A. 1982, Pis'ma Astron. Zh., 8, 612 (LS82)

Lutovinov, A., Tsygankov, S., Chernyakova, M. 2012, MNRAS, 423,1978

Masetti, N., Dal Fiume, D., Amati, L., Del Sordo, S., Frontera, F., Orlandini, M., \& Palazzi, E., 2004, A\&A, 423, 311

Mereghetti, S. 2008, A\&ARv, 15, 225

Meszaros, P., \& Nagel, W. 1985a, ApJ, 298, 147

Meszaros, P., \& Nagel, W. 1985b, ApJ, 299, 138

Morfill, G. E., Truemper, J., Tenorio-Tagle, G., \& Bodenheimer, P. 1984,

A\&A, 139, 7

Nagel, W. 1981, ApJ, 251, 278

Payne, D. G., \& Blandford, R. D., 1981, MNRAS, 196, 296

Rea, N., Esposito, P., Turolla, R., et al. 2010, Science, 330, 944

Reig, P., Torrejon, J. M., Neguerela, I., Blay, P., Ribo, M., \& Wilms, J., 2009, A\&A, 494, 1073

Reig, P., Torrejon, J. M., \& Blay, P. 2012, arXiv:1203.1490 
Romanova, M. M., Ustyugova, G. V., Koldoba, A. V., \& Lovelace, R. V. E. 2004, ApJ, 610, 920

Thompson, C., \& Duncan, R. C. 1995, MNRAS, 275, 255

Torrejon, J. M., Kreykenbohm, I., Orr, A., Titarchuk, L., \& Negueruela, I., 2004, A\&A, 423, 301

Trümper, J. E., Zezas, A, Ertan, Ü, \& Kylafis, N. D. 2010, A\&A, 518, A46 van Paradijs, J., Taam, R. E., \& van den Heuvel, E. 1995, A\&A, 299, L41
Wang, W., 2009, MNRAS, 398, 1428

Wang, W., 2010, A\&A, 520, 22

Wang, Z., Chakrabarty, D., \& Kaplan, D. L. 2006, Nature, 440, 772

White, N. E., Angelini, L., Ebisawa, K., et al. 1996, ApJ, 463, 83

Woods, P. M., \& Thompson, C. 2006, in "Compact Stellar X-ray Sources", eds. W.H.G. Lewin and M. van der Klis, Cambridge Univ. Press (astro-ph/0406133) 


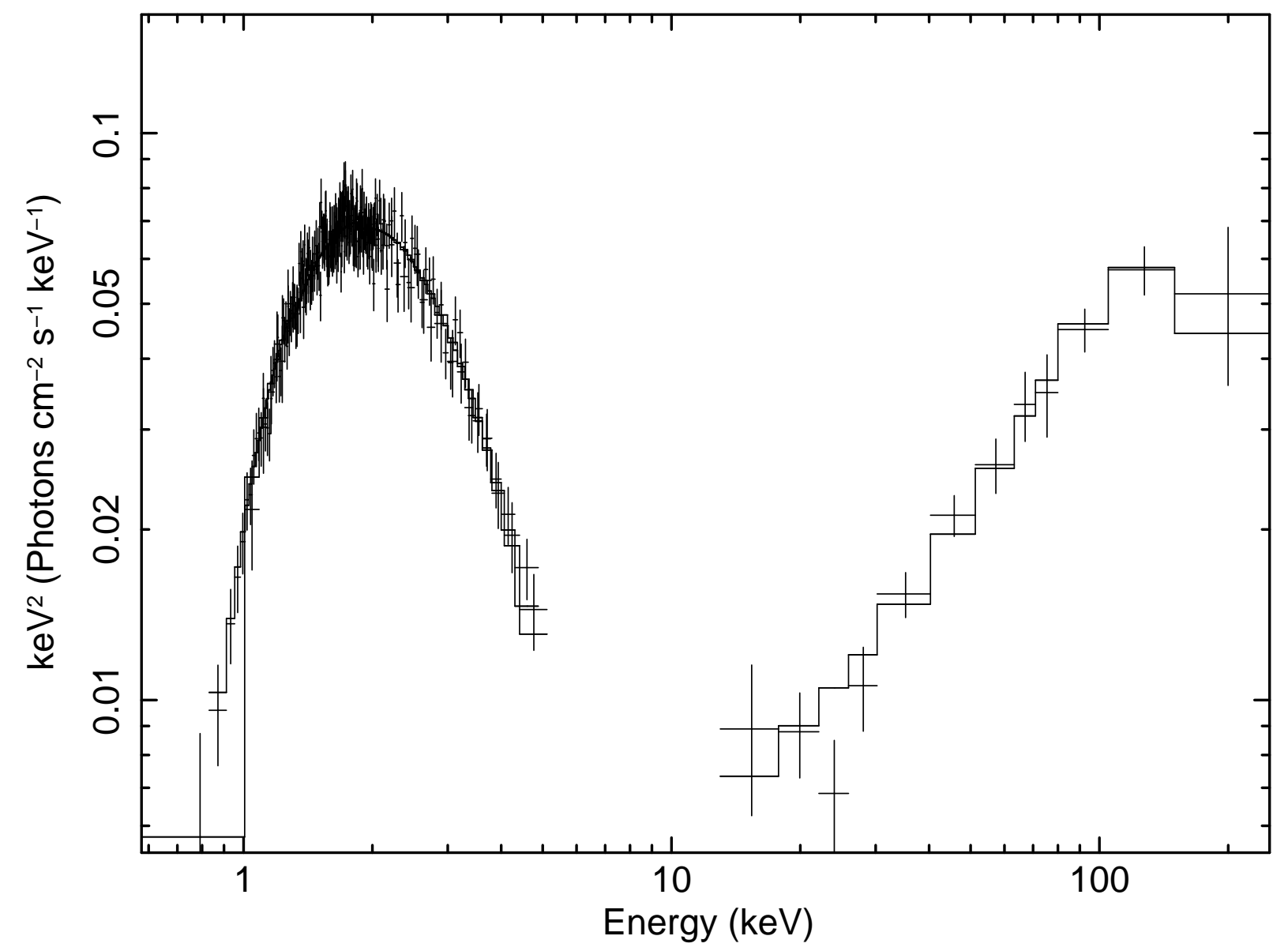

Figure 1. The best-fitting COMPTB model, along with the pulse phase averaged data from Chandra MEG and HEG $(<10 \mathrm{keV})$ and Integral ISGRI ( $>15 \mathrm{keV})$, c.f. Truemper et al. (2010. 

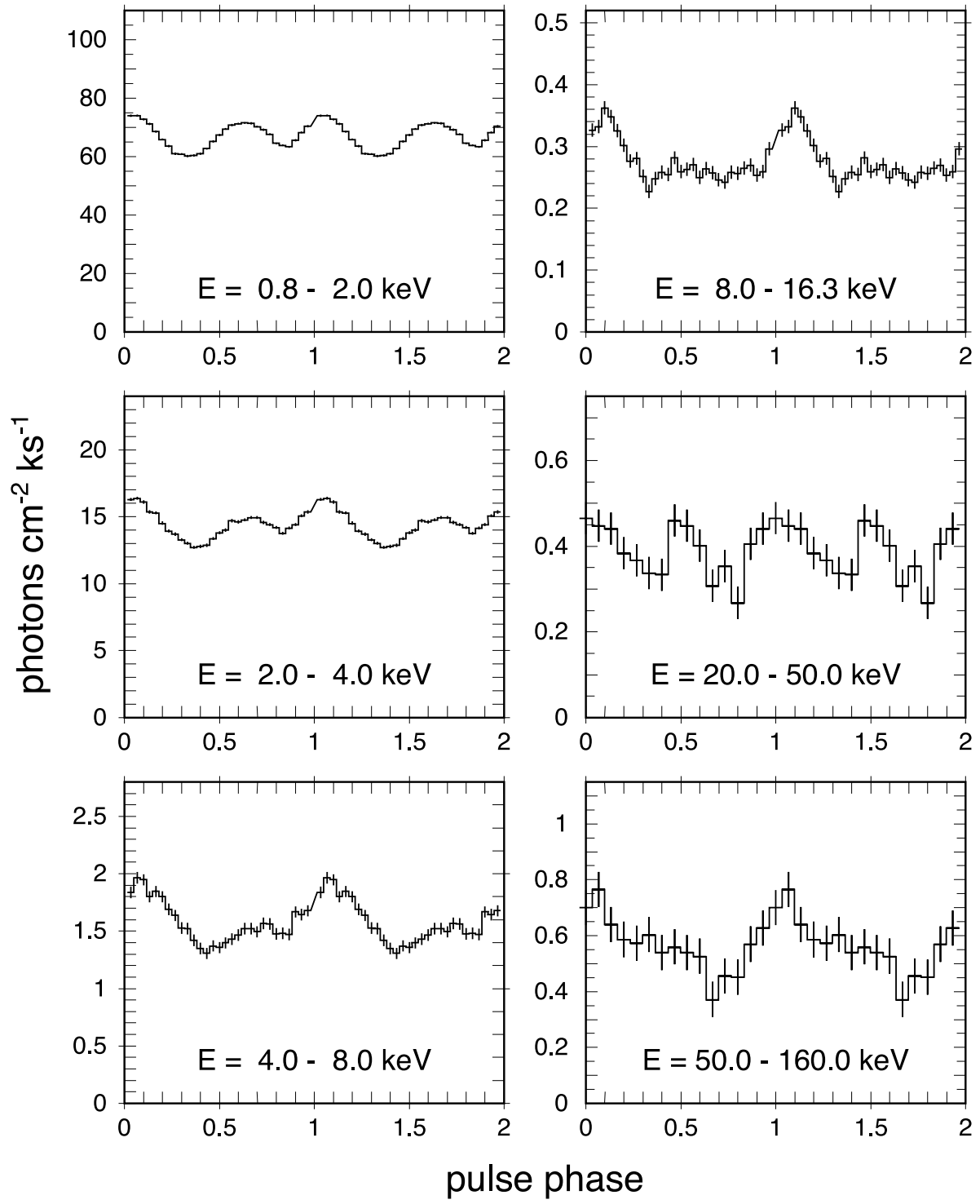

Figure 2. The histograms show the total pulse profiles (pulsed plus non-pulsed components) of $4 \mathrm{U} 0142+61$, derived from the observational data of den Hartog et al. (2008). 


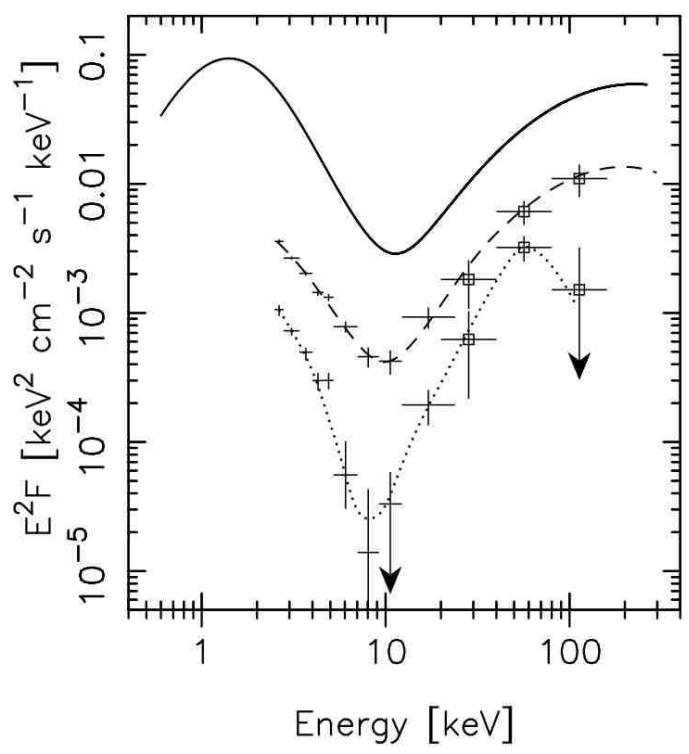

Figure 3. This Figure is taken from Fig. 8 of den Hartog et al. (2008). The black line (top one) represents the INTEGRAL/XMM-Newton total spectrum fit shown in that paper. The spectrum of the main pulse (in our model the fan beam), taken from the phase interval $0.85-0.35$, is shown as a dashed line). The spectrum of the secondary pulse (in our model the polar beam), taken from the phase interval $0.35-0.85$, is shown as a dotted line. The spectrum of the polar beam shows a steep rise up to a maximum at $\sim 60 \mathrm{keV}$. In our model, this bump is tentatively interpreted in terms of enhanced reflection due to cyclotron scattering of fan beam photons at the polar photosphere. 


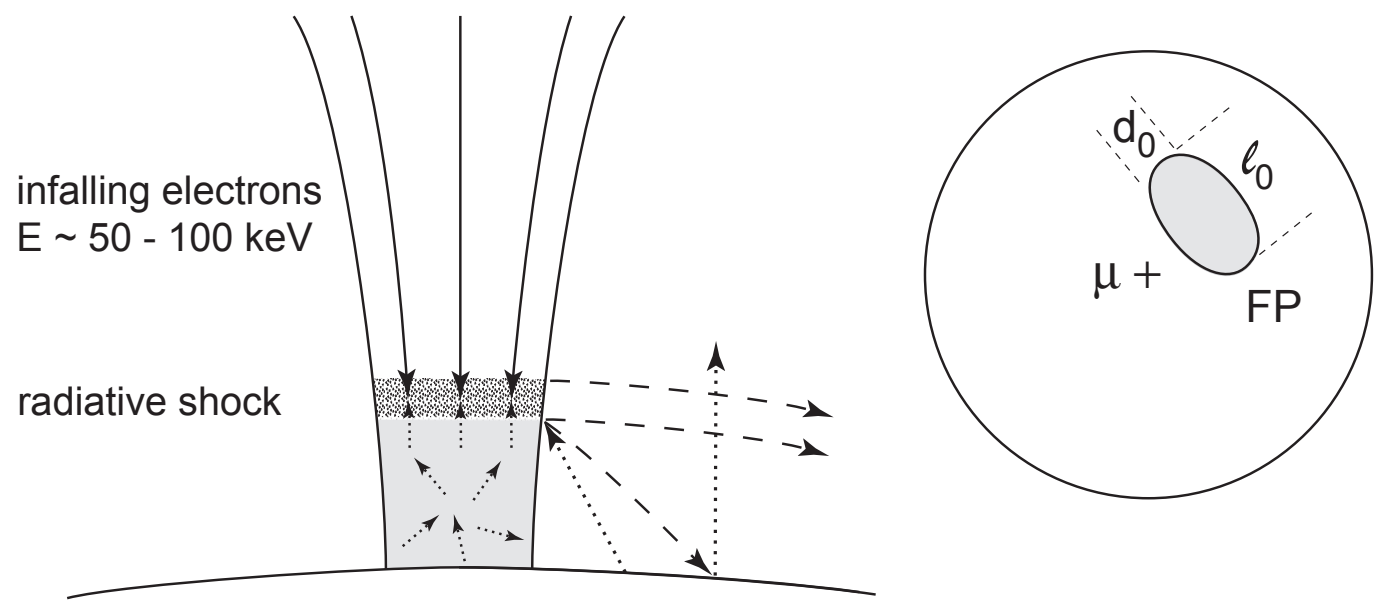

Figure 4. Fig. 4a (left): Schematic description of the beaming pattern. The different arrows show the mean direction of the respective component. Dashed arrows: Fan beam consisting of photons produced by thermal Comptonization $(E<10 \mathrm{keV})$ and bulk-motion Comptonization $(E>10 \mathrm{keV})$. It is subject to gravitational bending and part of the fan beam hits the neutron star photosphere, where it is scattered or absorbed. Dotted arrows: Polar beam consisting of fan beam photons after reflection (scattering) from the photosphere. Also thermal photons emitted from the hot photosphere contribute to the polar beam.

Fig. 4b (right): Footprint (FP) of the accretion column at the neutron star surface (see text). 

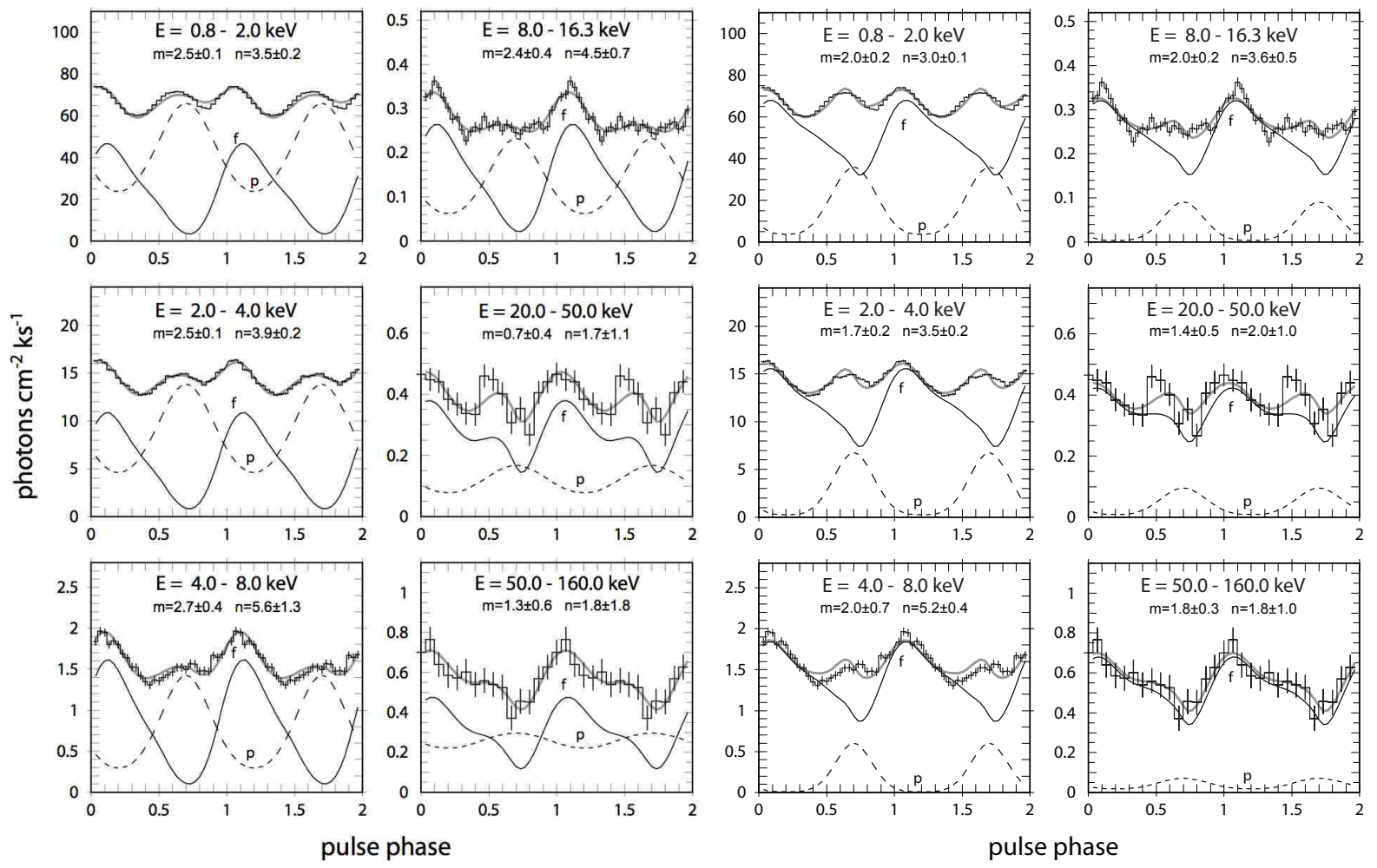

Figure 5. The black histograms show the total observed pulse profiles (pulsed plus non-pulsed components) of $4 \mathrm{U} 0142+61$ as given in Fig. 2 . The best-fit model for the pulse profiles is shown as a thick grey curve, in 5a for a height of the radiative shock at $H=0$, and in $5 \mathrm{~b}$ for $H=2 \mathrm{~km}$. The decomposition of the pulse profiles into the fan beam and the polar beam components are shown as solid and dashed lines, respectively. $m$ and $n$ are the exponents of the beaming functions of the fan beam and the polar beam, respectively (see text). 